\title{
5G NR CA-Polar Maximum Likelihood Decoding by GRAND
}

\author{
Ken R. Duffy*, Amit Solomon ${ }^{\dagger}$, Kishori M. Konwar ${ }^{\dagger}$ and Muriel Médard ${ }^{\dagger}$ \\ * Hamilton Institute, Maynooth University, Ireland. \\ $\dagger$ Research Laboratory of Electronics, Massachusetts Institute of Technology, U.S.A.
}

\begin{abstract}
CA-Polar codes have been selected for all control channel communications in 5 G NR, but accurate, computationally feasible decoders are still subject to development. Here we report the performance of a recently proposed class of optimally precise Maximum Likelihood (ML) decoders, GRAND, that can be used with any block-code. As published theoretical results indicate that GRAND is computationally efficient for shortlength, high-rate codes and 5G CA-Polar codes are in that class, here we consider GRAND's utility for decoding them. Simulation results indicate that decoding of $5 \mathrm{G}$ CA-Polar codes by GRAND, and a simple soft detection variant, is a practical possibility.

Index Terms-5G; CA-Polar Codes; GRAND
\end{abstract}

\section{INTRODUCTION}

Polar codes, which were introduced by E. Arikan in 2008, were the first explicit code construction to be provably channel capacity achieving [1], [2]. Their promise of high rates at short block-lengths led them to be considered for the protection of $5 \mathrm{G}$ control channel communications. Initial results, however, provided disappointing block error rate (BLER) performance [3], [4]. Subsequent work established that performance could be substantially enhanced by an additional layer of redundancy, where data is first coded with a Cyclic Redundancy Check (CRC) and then Polar coded, leading to CRC Assisted Polar (CA-Polar) codes. The standard decoding design is to first list-decode the Polar code, creating a collection of candidate code-words, and then select a decoded element by evaluating the CRC of each list member. This work has resulted in decoders that have shown significant improvements in BLER, particularly when availing of soft detection information [5], [4], [6], resulting in the 5G NR standard adopting CAPolar codes for all control communications.

The further development of accurate and computationally efficient CA-Polar decoders, particularly those that can work in the absence of soft information, is desirable. Since Shannon's earliest work [7] it has been known that Maximum Likelihood (ML) decoding is optimal in terms of accuracy for any code construction. In ML decoding, the decoded word is determined to be the most likely member of the code-book given the channel output. ML decoding has not been implemented in practice as it is not computationally feasible under existing proposals. Instead decoding algorithms are typically co-designed with particular code-book structures in mind and heuristically aim to approximately identify an ML decoding candidate.

An exception to this is the Guessing Random Additive Noise Decoding (GRAND) framework, which was introduced in 2018 [8] and works with any block-code. GRAND identifies an ML decoding, while GRANDAB (GRAND with Abandon- ment) [9], a variant with reduced computational complexity, either identifies an ML decoding or reports a decoding failure. Both have been theoretically proven to be capacity-achieving when used with random code-books [9]. In contrast to codebook oriented algorithms, GRAND and GRANDAB are noisecentric, and aim to infer the noise that has occurred on the channel from which the ML decoding can be deduced. They can decode CA-Polar codes in a single step, without the need for two separate docoders.

Unlike most of the more effective existing CA-Polar decoders, GRAND and GRANDAB are hard detection decoders that solely take demodulated symbols as input, potentially making them useful for hardware where soft detection information cannot be provided by the receiver to the decoder. If symbol reliability information, a simple form of soft information in which a symbol is marked to be either reliable or unreliable via, e.g., instantaneous SINR, is available, however, variants called SRGRAND and SRGRANDAB that avail of it to improve decoding accuracy and reduce computational complexity have recently been introduced [10], [11]. Mathematical results for random code-books suggest that GRAND and its variants are particularly appropriate for short, highrate codes [9], [11]. As in common target operating regimes, raw BER are sufficiently low to lend themselves to CA-Polar codes that are short-length and high-rates, the GRAND family of decoders seem to be promising candidates for 5G NR. Here we report simulation results on 5G CA-Polar decoding by the GRAND and SRGRAND approaches. These results suggest that accurate decoding $5 \mathrm{G}$ communications channel packets is practically feasible with GRANDAB and SRGRANDAB.

\section{Guessing Random Additive Noise Decoding}

The basis for GRAND is that for discrete channels subject to additive noise the following two algorithms produce the same output, but that the second one can be computationally feasible for high-rate codes while the first one is not. 1) ML decoding by brute force: given a received block of $n$ demodulated symbols, $y^{n}$, and a channel noise model, compute the likelihood that each code-word in the code-book, $c^{i, n}$, was transmitted given $y^{n}$ was received, and define the decoded element, $c^{*, n}$, to be one with the highest likelihood. 2) ML decoding by GRAND: taking noise sequences, $z^{n}$, in order from most likely to least likely based on the channel model, subtract them (in the Galois field of the symbols) from the received sequence $y^{n}$ and query if what remains, $y^{n} \ominus z^{n}$, is 


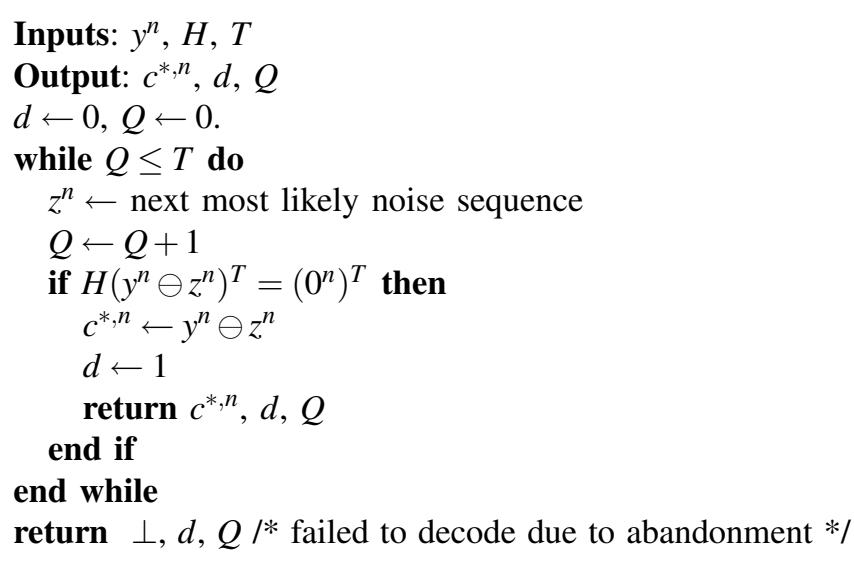

Fig. 1. Sketch of GRAND and GRANDAB. Given a channel output $y^{n}$, a block code's parity check matrix $H$, and a querying abandonment threshold $T\left(=\infty\right.$ for GRAND), if a code-book element $c^{*, n}$ is identified before a number of code-book queries, $Q$, corresponding to the guesswork threshold is exceeded, it is reported along with successful decoding, $d=1$; otherwise an abandonment failure is reported, $d=0$.

a member of the code-book, reporting the first instance, $c^{*, n}$, where that is true.

To GRAND, GRANDAB adds a counter for the number of noise guesses, i.e. code-book membership queries, made, and abandons guessing if more than a set number has been exceeded. It has been proven that GRANDAB is capacity achieving when used with random code-books so long as the abandonment threshold is set correctly [9].

For a linear code construction with parity check matrix $H$, one can test if a string, $y^{n}$, is a member of the code-book by a single matrix multiplication and comparison, $H\left(y^{n}\right)^{T} \stackrel{?}{=}\left(0^{n}\right)^{T}$. All 5G NR codes are linear and binary, so that the appropriate field is $\mathrm{GF}_{2}$ (i.e. mod 2). Control channel communications are first coded with a CRC, then interleaved if they are downlink communications, and finally Polar coded prior to modulation and transmission. All of those operations are linear, so that an input information word consisting of $k$ bits, $x^{k}$, is transformed into an $n$-bit code-word, $c^{n}$, by the linear map

$$
c^{n}=x^{k} G_{\mathrm{CRC}} M_{\text {Interleave }} G_{\text {Polar }},
$$

where $G_{\mathrm{CRC}}$ is the generator matrix for the CRC, $M_{\text {Interleave }}$ is the interleaving matrix (the identity for uplink communications), and $G_{\text {Polar }}$ is the generator for the Polar code.

As most decoders are code-book centric, two distinct decoders would normally need to be employed: one for the Polar Code and one for the CRC. As the GRAND algorithms are code-book agnostic, they can treat the CA-Polar code as a single linear code and decode both simultaneously by using the corresponding parity check matrix $H_{\mathrm{CA}-\text { Polar }}$, and testing code-book membership, $H_{\text {CA-Polar }}\left(y^{n}\right)^{T} \stackrel{?}{=}\left(0^{n}\right)^{T}$. As a result, the decoder provides a code-word that is consistent with both the CRC and Polar code without the need for two separate decoders. Pseudo-code for GRANDAB can be found in Fig. 1.

\section{5G CA-PolAR CODES BY GRAND}

Core to GRAND's accuracy is querying noise sequences in order from most likely to least likely. An interleaved transmission corresponds to a Binary Symmetric Channel (BSC) [12] where the binary channel output $y^{n}$ can be written as the binary input code-word, $c^{n}$, plus independently binary additive channel noise $z^{n}, y^{n}=c^{n} \oplus z^{n}$. Thus we use the likelihood ordering determined by the BSC where the most likely sequence is all zeros, followed by each of those with one bit flip in any order, followed by those with two bit flips in any order, etc.

In our implementation of the GRANDAB, we abandon guessing if no element of the code-book has been identified after testing all sequences with up to $\mathrm{AB}$ bit flips. If the noise introduces more than $\mathrm{AB}$ bit flips, as no such error sequences will be queried, this necessarily results in an error either due to abandonment or an erroneous decoding. Using GRAND, we first establish that an appropriate value of $\mathrm{AB}$ is solely a function of the code-book and not channel conditions.

An example high rate CA-Polar code in the 5G NR standard is for the uplink where $k=105$ information bits are converted results into $n=128$ transmitted bits per block, giving a rate of 0.82 . Fig. 2 (a) plots the cumulative distribution of the number of code-book queries, $Q$, made by GRAND until a decoding is identified conditioned on the number of bit flips due to noise. For each number of conditioning bit flips, $b$, these plots were created by simulation of a large number of noise sequences with $b$ bit flips randomly located in $1, \ldots, n$. For $b=1,2,3$, these distributions have similar structure as they primarily correspond to the number of code-book queries until a correct decoding is identified. When $b=4$, the distribution looks distinct because many of the decodings are erroneous.

Also shown in the legend of Fig. 2 (a) is GRAND's BLER conditioned on that number of bit flips. As GRAND identifies an ML decoding, a more accurate decoding algorithm is not possible with only hard detection information. If a single bit is flipped, the conditional BLER is zero and the decoding is always correct. If four bits are flipped, however, the ML decoding is in error for approximately $65 \%$ of ML decodings as identified by GRAND, indicating that, with high probability, the minimum distance of the code is 3 or 4 . In a concatenated code design with an optimial outer code, the additional redundancy required to correct an erroneous decoding is just over twice that required to correct an erasure [13]. As a result, if, for a given number of bit flips, the ML decoding has a conditional BLER $>33 \%$ of decodings, the decoder would be better off not doing the work required to determine the ML decoding, but instead report an erasure by abandoning via GRANDAB. By this rationale, for the CA-Polar $[128,105]$ code, GRANDAB should abandon decoding if no code-book element is identified after up to all Hamming weight sequences of $\mathrm{AB}=3$ have been queried.

From the data contained in Fig. 2, we can efficiently determine GRANDAB's BPSK AWGN BLER and complexity, in terms of the number of code-book queries, via importance 

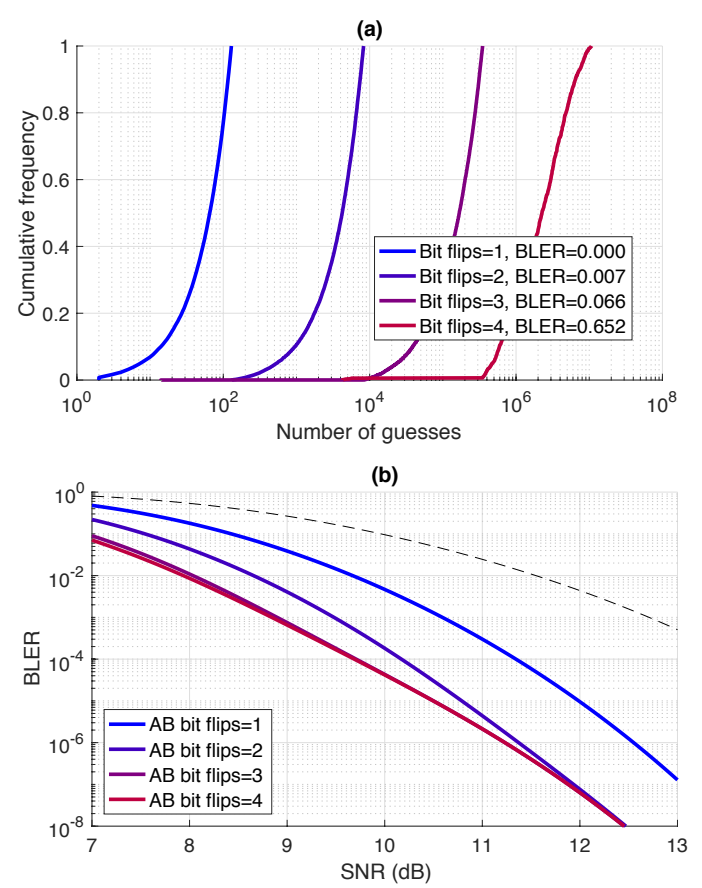

(c)

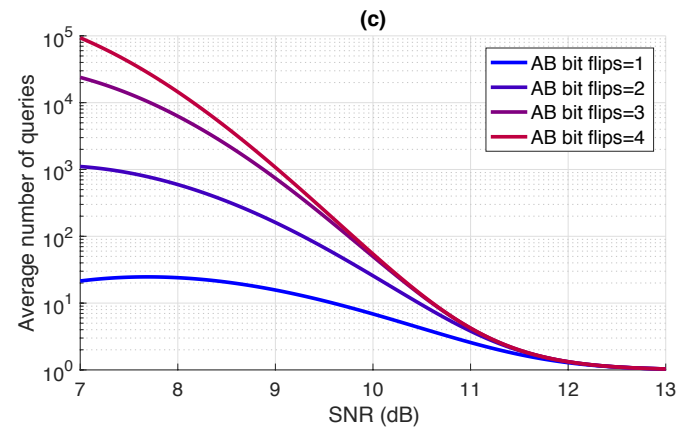

Fig. 2. 5G NR uplink, 11-bit CRC, CA-Polar [128,105] decoding by GRAND and GRANDAB. (a) Conditioned on the number of bits flips, the cumulative distribution of how many code-book queries are made before a decoding is identified. The legend reports the block error rate given that number of bit flips. (b) GRANDAB BLER vs SNR in an AWGN BPSK channel. The dashed black line indicates uncoded block error rate for a 128 bit transmission. The solid lines indicate BLER for GRANDAB given the abandonment after querying all noise sequences with weight up to AB. (c) Average number of code-book queries vs SNR until a decoding is found or GRANDAB abandons guessing.

sampling, the law of total probability, and the law of total expectation. That is, with $B$ being the number of bit flips due to noise, we have

$$
\begin{aligned}
\mathrm{BLER} & =\sum_{b=0}^{\mathrm{AB}} P(\text { error } \mid B=b) P(B=b)+P(B>\mathrm{AB}) \\
E(Q) & =\sum_{b=0}^{\mathrm{AB}} E(Q \mid B=b) P(B=b)+\left(\sum_{b=0}^{\mathrm{AB}}\left(\begin{array}{l}
n \\
b
\end{array}\right)\right) P(B>\mathrm{AB}) .
\end{aligned}
$$

Assuming binary phase shift keying (BPSK) to $\{-1,+1\}$, and AWGN with variance $\sigma^{2}$, the signal-to-noise ratio is $\mathrm{SNR}=-10 \log _{10} \sigma^{2}$ in $\mathrm{dB}$ (note that there are other definitions of SNR, e.g. Eb/N0, which may complicate direct comparison) and the probability of a bit flip is $p=P(\sigma \mathscr{N}>1)$, where $\mathscr{N}$ denotes a normal random variable with mean 0 and variance 1 . $B$ is distributed as a binomial random variable with probability $p$ on $n$ trials, where $n$ is the code-length. Thus to evaluate the BLER and the computational complexity, we use the conditional information in Fig. 2 (a) to estimate $P(\operatorname{error} \mid B=b)$ and $E(Q \mid B=b)$, and then use eq. (1).

GRANDAB's BLER vs SNR is shown in Fig. 2 (b). The dashed black line indicates the uncoded BLER (i.e. the probability that a single bit in a 128 bit block is flipped by noise), while the colored lines correspond to GRANDAB with a range of abandonment weights. Consistent with earlier logic, the BLER of GRANDAB with $\mathrm{AB}=3$ or 4 is essentially identical, indicating that there is no gain in BLER to be had with this CA-Polar code by seeking a decoding where four or more bits have been flipped. In comparison to abandonment after only one or two bit flips, $\mathrm{AB}=1$ or $\mathrm{AB}=2$, however, both have substantially better BLER. This reflects the fact that with $\mathrm{AB}=3$, GRANDAB is essentially providing all of the merits of an ML decoder, but with an a priori upper bound on decoding complexity.

In terms of complexity, Fig. 2 (c) reports the average number of code-book queries until GRANDAB identifies a decoding. A standard operational regime would seek a BLER of $<10^{-3}$, which is achieved with this code for a SNR of $9 \mathrm{~dB}$ or higher whereupon it is over two orders of magnitude less than the uncoded BLER. For those SNRs, GRANDAB with $\mathrm{AB}=3$ performs fewer than $10^{3}$ code-book queries per decoding on average, i.e. $<10$ code-book queries per decoded bit. As codebook queries can readily be parallelized, this holds significant promise for both software and hardware implementations.

For comparison, another high rate CA-Polar code encodes $k=99$ information bits into $n=128$ bits for the downlink, giving a rate of 0.77 . Fig. 3 plots analogous results to those of the $[128,105]$ uplink code shown in Fig. 2. In order to fully utilize the redundancy available in this lower-rate code, the abandonment has to be pushed out to a Hamming weight of $\mathrm{AB}=4$, at a cost of extra work for the decoder. This potentially counter-intuitive result reflects that the GRAND approach favors high rate codes, which is a consequence of the fact that the complexity of GRAND algorithms can only decrease as code-rates increase.

These results suggest that GRANDAB holds promise as an accurate and efficient hard-detection CA-Polar decoder. If in addition quantized symbol reliability information can be provided by the receiver to the decoder, recent theoretical results suggest that further improvements in BLER and decreases in complexity are possible [10], [11], which we now explore.

\section{5G NR CA-Polar COdes By SRGRAND}

In BPSK, each coded bit is communicated via the phase of a continuous wave that is impacted by noise before reception and demodulation. Fig. 4) provides a heat-map plot of the probability density of the received signal as a function of the SNR in an AWGN model. In the hard detection setting of the previous section, the received signal is demodulated to a 0 or 1 depending on whether it is to the left or right of the 


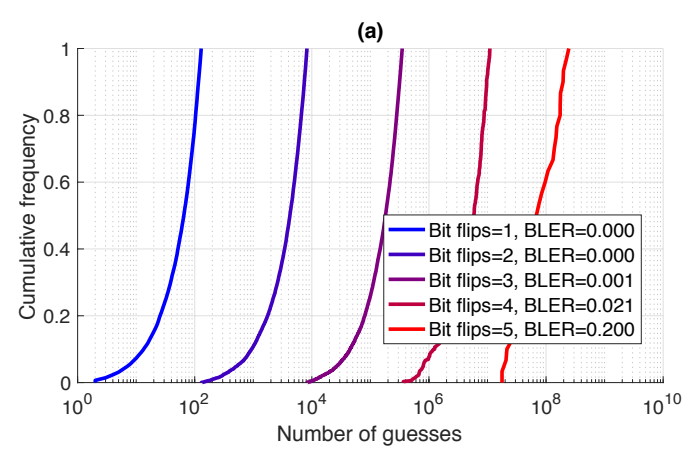

(b)

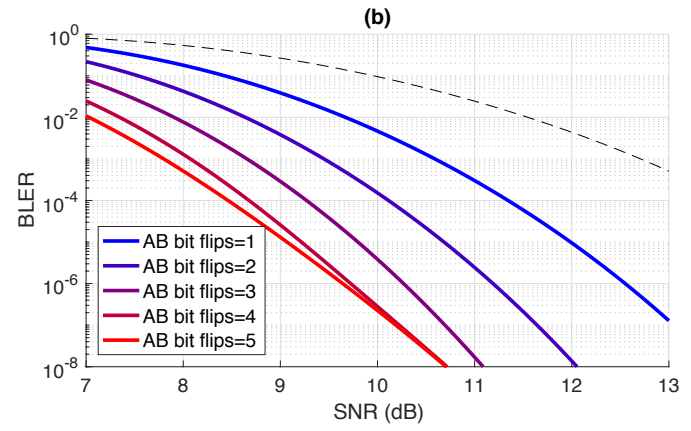

(c)

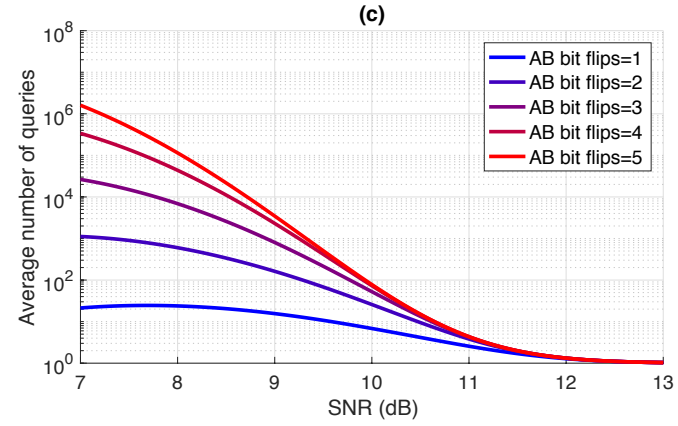

Fig. 3. 5G NR downlink, 24-bit CRC, CA-Polar [128,99]. (a-c) As in Fig. 2.

vertical dashed black line, and the resulting bits passed to the decoder. Hard detection decoders execute solely based on that information.

Soft detection decoders attempt make further use of the received signal to better inform their decoding, e.g. [14], [15], [16], [17], [18]. Incorporating soft detection information results in improved accuracy, but typically at the cost of increased computational complexity in the decoding process. Moreover, provision of soft detection information, such as instantaneous SINR, by a receiver to a decoder requires the passing of real-valued data, which is costly in terms of memory and I/O. An alternative approach, first considered within Chase decoding [19], [20], is for the receiver to quantize the received signal and provide symbol reliability information where, in addition to the hard-demodulated symbols, $y^{n}$, the receiver passes a binary string, $s^{n}$, that labels symbols as reliable or unreliable. Requiring only one additional bit per received symbols, symbol reliability information is much less costly to provide to the decoder.

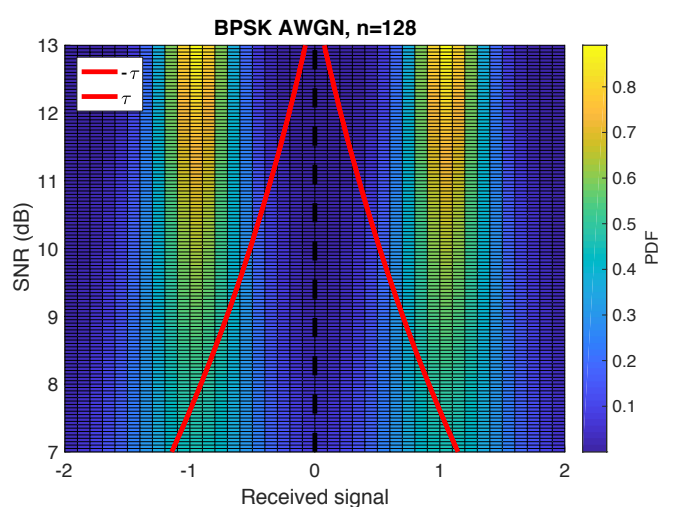

Fig. 4. BPSK AWGN symbol reliability. Bits are modulated to $\{-1,+1\}$ and are subject to AWGN. Shown is a heat map of the probability density of the received signal (x-axis) as a function of SNR (y-axis). The codebook independent quantization of soft detection information in SRGRAND marks bits corresponding to signals received outside of the red region as reliable, while those that within are unreliable. The region is chosen so that the probability that any bit in a transmitted $n=128$ block is marked as reliable in error (e.g. the received signal is left of the red-line, while a +1 was transmitted) has probability MERR $=10^{-4}$.

Inputs: $y^{n}, H, T, s^{n}$,

Output: $c^{*, n}, d, Q$

$d \leftarrow 0, Q \leftarrow 0$.

while $Q \leq T$ do

$z^{n} \leftarrow$ zeros where $s^{n}$ is zero and the next most likely noise sequence mapped to the ones of $s^{n}$

$Q \leftarrow Q+1$

$$
\begin{aligned}
& \text { if } H\left(y^{n} \ominus z^{n}\right)^{T}=\left(0^{n}\right)^{T} \text { then } \\
& c^{*, n} \leftarrow y^{n} \ominus z^{n} \\
& d \leftarrow 1
\end{aligned}
$$

return $c^{*, n}, d, Q$

end if

end while

return $\perp, d, Q / *$ failed to decode due to abandonment */

Fig. 5. Sketch of SRGRAND and SRGRANDAB. Given the inputs in Fig. 1 and $s^{n}$, a binary mask of length $n$ with $l^{n}$ ones in locations indicating unreliable symbols, if a code-book element $c^{*, n}$ is identified before a a guesswork threshold is exceeded it is reported along with successful decoding, $d=1$; otherwise an abandonment failure is reported, $d=0$.

SRGRAND and SRGRANDAB [10], [11] expect such symbol reliability information. For BPSK, we created that information by setting a threshold for the received signal, $\pm \tau$, above or below which one is confident that the transmitted phase was \pm 1 . We define the Mask Error Rate, MERR, to be error rate due to erroneously marking one or more bits in a block as reliable when they are not. The threshold, $\tau$, is determined as a function of the SNR, the code-length, and MERR. As the probability a bit is erroneously labeled as reliable when it is incorrect is $P(\sigma \mathscr{N}>1+\tau)$, we have MERR $=1-P(\sigma \mathscr{N} \leq$ $1+\tau)^{n}$. Hence we set $\tau=\sigma\left(F_{\mathscr{N}}^{-1}\left((1-\mathrm{MERR})^{1 / n}\right)-1\right)$, where $F_{\mathscr{N}}^{-1}$ is the inverse of a Normal distribution. For $n=128$ and MERR $=10^{-4}$, the red lines in Fig. 4 illustrate the masked region as a function of SNR. 
Armed with this symbol reliability information, pseudo code for SRGRANDAB is given in Fig. 5. SRGRANDAB proceeds as GRANDAB, but with subtraction of noise sequences restricted to only bits marked as unreliable. The result is a more targeted querying on an effectively smaller code, leading to better decoding accuracy and less complexity. As with the hard detection decoders, BLER and complexity performance can be determined by the importance sampling approach, first conditioning on mask-lengths (i.e. number of unreliable bits) and then on the number of bit flips. In the BPSK AWGN setting, the probability that a bit is marked as unreliable is $q=P(\sigma \mathscr{N} \leq-1+\tau)-P(\sigma \mathscr{N} \leq-1-\tau)$, while the conditional probability that a bit is flipped given it is marked as unreliable is $p=(P(\sigma \mathscr{N} \leq 1+\tau))-P(\sigma \mathscr{N}(0,1) \leq 1) / q$.
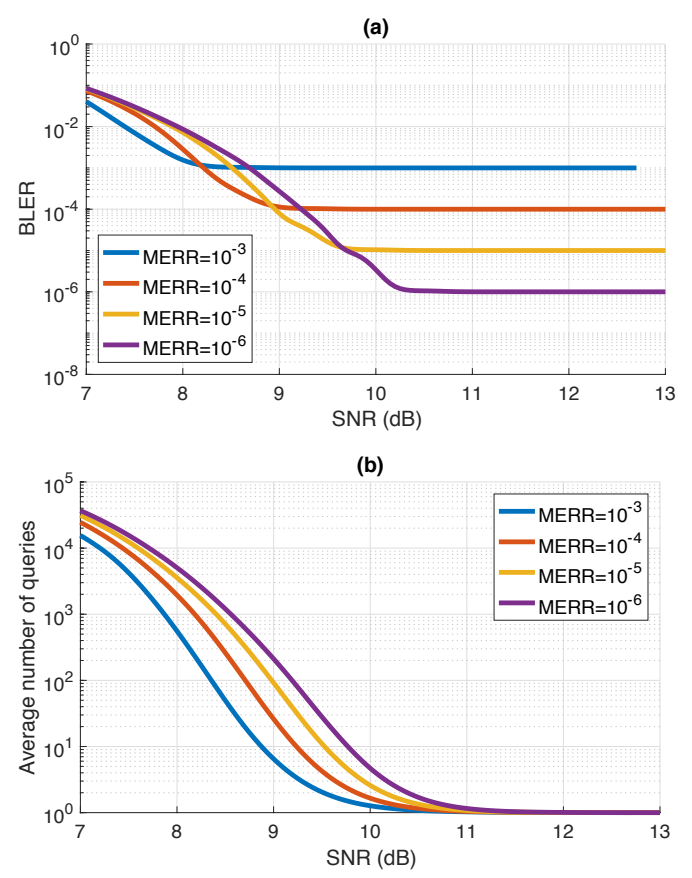

Fig. 6. 5G NR CA-Polar uplink $[128,105]$ decoding by SRGRANDAB. For different values of target mask error rate, MERR, accuracy and complexity, where the guesswork threshold is set so as to allow all three bit flip sequences be queried for a mask of 128 bits. (a) BLER vs SNR. (b) Average number of code-book queries until a decoding is identified or SRGRANDAB abandons guessing.

For the $[128,105]$ CA-Polar code considered previously, Fig. 6 reports the performance of SRGRANDAB where the guesswork threshold is set to allow the querying of all three bit flip noise sequences for a mask of 128 bits. For a range of values of MERR, the target mask-error probability, Fig. 6 (a) reports BLER vs SNR. With a MERR $=10^{-4}$, a BLER of $10^{-4}$ is achieved from a SNR of 9 for SRGRANDAB, in comparison to a SNR of 9.75 for GRANDAB as provided in Fig. 3 (b). As well as a gain in decoding accuracy, SRGRANDAB provides a significant reduction in decoding complexity due to the targeted nature of its querying where at an SNR of 9 for the target MERR $=10^{-4}$, this BLER is achieved with fewer than 12 code-book queries per decoding on average, in comparison to 110 for the hard detection equivalent.

\section{Conclusions}

Due to their high rates at short code-lengths, CA-Polar codes have been selected for all control channel communications in 5G NR. Current designs see list decoding, typically with soft detection information, of the Polar code followed by use of the CRC to select a decoding. Here we have established that the hard detection algorithms GRAND and GRANDAB, which identify optimal ML decodings and decode both codes in a single step, offer a viable alternative method. If a receiver can pass symbol reliability information to the decoder, use of SRGRANDAB can increase decoding precision with reduced computational complexity. Taken together, the GRAND approach seems promising for use with high-rate 5G NR CAPolar codes. Further work may consider ancillary issues such as rate matching and recovery, or further use of more detailed soft detection information.

\section{REFERENCES}

[1] E. Arikan, "Channel polarization: A method for constructing capacityachieving codes," in IEEE Int. Symp. Inf. Theory, 2008, pp. 1173-1177.

[2] — "Channel polarization: A method for constructing capacityachieving codes for symmetric binary-input memoryless channels," IEEE Trans. Inform. Theory, vol. 55, no. 7, pp. 3051-3073, 2009.

[3] H. D. Pfister, "A brief introduction to Polar codes," Supplemental Material for Advanced Channel Coding, 2014.

[4] I. Tal and A. Vardy, "List decoding of Polar codes," IEEE Trans. Inf. Theory, vol. 61, no. 5, pp. 2213-2226, 2015.

[5] K. Niu and K. Chen, "CRC-aided decoding of Polar codes," IEEE Commun. Letters, vol. 16, no. 10, pp. 1668-1671, 2012.

[6] A. Balatsoukas-Stimming, M. B. Parizi, and A. Burg, "LLR-based successive cancellation list decoding of Polar codes," IEEE Trans. Signal Process., vol. 63, no. 19, pp. 5165-5179, 2015.

[7] C. E. Shannon, "A Mathematical Theory of Communication," Bell Syst. Tech. J., vol. 27, pp. 379-423, 623-656, 1948.

[8] K. R. Duffy, J. Li, and M. Médard, "Guessing noise, not code-words," in IEEE Int. Symp. on Inf. Theory, 2018.

[9] — "Capacity-achieving guessing random additive noise decoding (GRAND)," IEEE Trans. Inf. Theory, vol. 65, no. 7, pp. 4023-4040, 2019.

[10] K. R. Duffy and M. Médard, "Guessing random additive noise decoding with soft detection symbol reliability information," in IEEE Int. Symp. on Inf. Theory, 2019.

[11] _ _ "Guessing random additive noise decoding with soft detection symbol reliability information (SRGRAND)," arXiv:1902.03796, Tech. Rep., 2019.

[12] C. Heegard and S. B. Wicker, "Interleaving," in Turbo Coding. Springer, 1999, pp. 35-63.

[13] R. Roth, Introduction to coding theory. CUP, 2006.

[14] A. B. Cooper, "Soft decision decoding of block codes," U.S. Army Laboratory Command, Maryland, Tech. Rep., 1988.

[15] L. Hanzo, T. H. Liew, and B. L. Yeap, Soft Decoding and Performance of $B C H$ Codes. IEEE, 2002, pp. 75-103.

[16] T. Kaneko, T. Nishijima, and S. Hirasawa, "An improvement of softdecision maximum-likelihood decoding algorithm using hard-decision bounded-distance decoding," IEEE Tran. Inf. Theory, vol. 43, no. 4, pp. 1314-1319, 1997.

[17] V. Guruswami and M. Sudan, "Improved decoding of Reed-Solomon and algebraic-geometry codes," IEEE Tran. Inf. Theory, vol. 45, no. 6, pp. 1757-1767, 1999.

[18] J. Xing, L. Chen, and M. Bossert, "Algebraic soft decoding algorithms for Reed-Solomon codes using module," CoRR, vol. 1712.00938, 2017.

[19] D. Chase, "Class of algorithms for decoding block codes with channel measurement information," IEEE Tran. Inf. Theory, vol. 18, no. 1, pp. 170-182, 1972.

[20] H. Mani and S. Hemati, "Symbolic stochastic Chase decoding of ReedSolomon and BCH codes," CoRR, vol. 1707.04390, 2017. 